\title{
Silica-supported silver nanoparticles: Tailoring of structure-property relationships
}

\author{
Davide Barreca ${ }^{\text {a) }}$ \\ Instituto di Scienze e Tecnologie Molecolari-Consiglio Nazionale delle Richerche (ISTM-CNR) \\ and Consorzio Interuniversitario Nazionale per la Scienza e Tecnologia dei Materiali (INSTM), \\ Department of Chemical Sciences, Padova University, Via Marzolo, 1-35131 Padova, Italy \\ Alberto Gasparotto, Cinzia Maragno, and Eugenio Tondello \\ Department of Chemical Sciences, Padova University and Consorzio Interuniversitario Nazionale per la \\ Scienza e Tecnologia dei Materiali (INSTM), Via Marzolo, 1-35131 Padova, Italy \\ Stefano Gialanella \\ Department of Materials Engineering and Industrial Technologies, Trento University, Via Mesiano, \\ 77-38050 Trento, Italy
}

(Received 27 September 2004; accepted 14 December 2004; published online 16 February 2005)

\begin{abstract}
Silica-supported silver nanoparticles were obtained by rf sputtering from Ar plasmas under soft synthesis conditions, with particular attention to the combined influence of rf power and total pressure on the system composition, nanostructure, morphology, and optical properties. In order to attain a thorough insight into the nucleation and growth phenomena of Ag nanoparticles on the silica substrate, several in situ and ex situ characterization techniques were used. In particular, a laser reflection interferometry system was employed for a real-time monitoring of the deposition process, providing useful and complementary information with respect to the other ex situ techniques (x-ray photoelectron spectroscopy and x-ray excited Auger electron spectroscopy, glancing incidence x-ray diffraction, atomic force microscopy, optical-absorption spectroscopy, and transmission electron microscopy). The above investigations evidenced the formation of silver-based nanosystems (average crystallite size $\leqslant 10 \mathrm{~nm}$ ), whose features (metal content, Ag particle size and shape, structure and optical properties) could be carefully tailored by moderate and controlled variations of the synthesis parameters. (C) 2005 American Institute of Physics. [DOI: 10.1063/1.1856213]
\end{abstract}

\section{INTRODUCTION}

Nanocomposite materials based on silver nanoparticles supported and/or embedded on/in various matrices ${ }^{1-10}$ have been widely investigated for their appealing chemical and physical properties. Among the possible applications, great interest has been devoted to their use in advanced technological fields including heterogeneous catalysis, ${ }^{2-4,10-13}$ gas sensing, ${ }^{1,14,15}$ optics, and optoelectronics. ${ }^{6,8,16-19}$ As a general rule, material features are strongly dependent on silver nanoparticle dimensions, size distribution and shape, as well as on the Ag amount and its dispersion. ${ }^{4,8,15,20,21}$ Further characteristics influencing the system properties are the chemical nature of the supporting/embedding matrix and its structural and morphological characteristics. ${ }^{1,13,20,22}$ In fact, the mutual interactions between silver nanoparticles and their surroundings ${ }^{5}$ result in peculiar features that are not displayed by the metal alone. ${ }^{20-25}$ Although the possibility of tailoring material properties represents a major advantage for its applications, it often constitutes a drawback from a synthetic point of view, since it requires the use of suitable preparation and processing techniques to obtain the desired functional performances. ${ }^{26}$

In particular, as concerns $\mathrm{Ag} / \mathrm{SiO}_{2}$ nanosystems, many synthesis routes have been employed including sol-gel and

\footnotetext{
${ }^{\text {a) }}$ Author to whom correspondence should be addressed; FAX: +39-498275161; electronic mail: davide@chin.unipd.it
}

impregnation, ${ }^{1,13,16,24}$ ion implantation, ${ }^{27,28}$ evaporation, ${ }^{4,29,30}$ combined thermal and electron-beam deposition, ${ }^{31}$ and sputtering. ${ }^{19,25,32}$ Most of the above techniques typically require the use of drastic preparation and/or treatment conditions, thus resulting in an expensive and/or difficult process control. Moreover, despite the extensive research activity on silver-based nanosystems, literature studies have often described the synthesis of $\mathrm{Ag} / \mathrm{SiO}_{2}$ materials with a high metal volume fraction, while the early nucleation stages of $\mathrm{Ag}$ on silica have not been thoroughly investigated up to date. In this context, the possibility of probing the system characteristics by a multitechnique approach involving both in situ and ex situ analysis methods plays a crucial role.

In this work, silica-supported silver nanoparticles have been obtained by rf sputtering of Ag from Ar plasmas, with particular regard to the first nucleation stages and to the property evolution as a function of the metal content. Attention has mainly been devoted to the use of soft processing conditions, focusing on the influence of the applied rf power and the total pressure on the system features in terms of chemical composition, surface morphology, nanostructure, and optical properties.

\section{EXPERIMENT}

\section{A. Synthesis}

Silver depositions were performed on commercial silica slides (Heraeus, Quarzschmelze, Hanau, Germany) by a two- 
TABLE I. Synthesis conditions for $\mathrm{Ag} / \mathrm{SiO}_{2}$ nanosystems. In all cases, the sputtering time and deposition temperature corresponded to $10 \mathrm{~min}$ and $60{ }^{\circ} \mathrm{C}$, respectively. $p$ and $V_{\text {bias }}$ denote the total pressure and self-bias potential, respectively.

\begin{tabular}{ccccc}
\hline \hline & Sample & $\begin{array}{c}\text { rf power } \\
(\mathrm{W})\end{array}$ & $\begin{array}{c}p \\
(\mathrm{mbar})\end{array}$ & $\begin{array}{c}V_{\text {bias }} \\
(\mathrm{V})\end{array}$ \\
\hline \multirow{2}{*}{ Set 1 } & 1 & 5 & 0.38 & -270 \\
& 2 & 10 & & -372 \\
& 3 & 15 & & -442 \\
& 4 & 25 & & -543 \\
Set 2 & 5 & 5 & 0.23 & -281 \\
& 6 & 10 & & -376 \\
& 7 & 15 & & -448 \\
& 8 & 25 & & -560 \\
Set 3 & & & & \\
& 9 & 5 & 0.08 & -301 \\
& 10 & 10 & & -388 \\
& 11 & 15 & & -460 \\
& 12 & 25 & & -574 \\
\hline \hline
\end{tabular}

electrode custom-built rf-sputtering apparatus $(\nu$ $=13.56 \mathrm{MHz})^{33}$ using electronic-grade Ar as feed gas and a 2-in.-diameter Ag target (0.1 mm thick; BAL-TEC AG, 99.99\%) as silver source. During each experiment, two substrates were mounted on the resistively heated electrode, using an electrode-to-electrode distance of $50 \mathrm{~mm}$. In order to investigate the influence of plasma parameters on the nanosystem features, particularly soft conditions were adopted. To this aim, depositions were carried out at a substrate temperature of $60{ }^{\circ} \mathrm{C}$ to minimize the influence of thermal effects. ${ }^{4}$ After the optimization of the synthesis parameters, three sample sets were prepared (see Table I) using different combinations of rf power $(W)$ and total pressure $(p)$, yielding, in turn, different self-bias potentials ( $V_{\text {bias}}$, see below). Special precautions were taken in order to minimize the exposure of silver-based nanosystems to the outer atmosphere. To this aim, at the end of the deposition, samples were immediately transferred to a desiccator evacuated by a rotary pump and subsequently backfilled with nitrogen. Conversely, when specimens were exposed to the outer atmosphere, a progressive oxidation was evidenced after a few days of aging, in agreement with previous reports. ${ }^{16}$ All obtained samples were characterized without any further ex situ treatment.

\section{B. Characterization}

Laser reflection interferometry (LRI) measurements were carried out using a custom-built apparatus, consisting of a He-Ne diode laser $(\lambda=670 \mathrm{~nm})$ incident on the substrate at an angle of $70^{\circ}$ from the normal. The light reflected by the growth surface was collected by a $p-i-n$ diode and the signal was digitized and recorded versus time by a computer. In order to avoid undesired interferences from external light and Ar plasma emission lines, ${ }^{34}$ a filter centered at $670 \mathrm{~nm}$ with a $10-\mathrm{nm}$ bandwidth $^{35}$ was mounted in front of the detector diode. Silica slides used for LRI measurements were ground on the back surface to remove undesired reflections from the substrate/electrode interface.
X-ray photoelectron spectroscopy (XPS) and x-ray excited Auger electron spectroscopy (XE-AES) measurements were run on a Perkin Elmer $\Phi$ 5600ci spectrometer at pressures lower than $10^{-9}$ mbar, using a monochromatized Al $K \alpha$ excitation source $(1486.6 \mathrm{eV})$. Binding-energy (BE) correction was performed assigning to the $\mathrm{C} 1 \mathrm{~s}$ line of adventitious carbon a value of $284.8 \mathrm{eV}$. The estimated BEs standard deviation corresponded to $\pm 0.2 \mathrm{eV}$. After a Shirley-type background subtraction, the raw spectra were fitted using a nonlinear least-squares deconvolution program. The atomic compositions were evaluated using sensitivity factors provided by $\Phi V 5.4 A$ software. Sputtering treatments were performed using an $\mathrm{Ar}^{+}$beam at $2.5 \mathrm{kV}$ with an argon partial pressure of $5 \times 10^{-8}$ mbar and a rastered area of $2 \times 2 \mathrm{~mm}^{2}$.

Glancing incidence $\mathrm{x}$-ray diffraction (GIXRD) patterns were recorded by a Bruker D8 Advance diffractometer equipped with a Göbel mirror and a $\mathrm{Cu} K \alpha$ source $(40 \mathrm{kV}, 40$ $\mathrm{mA}$ ), at a fixed incidence angle of $1.5^{\circ}$. The average $\mathrm{Ag}$ crystallite size was estimated by means of the Scherrer equation.

Atomic force microscopy (AFM) micrographs were recorded by a Park Autoprobe CP instrument operating in contact mode in air. Background subtraction was performed using the PROSCAN 1.3 software from Park Scientific. Images were taken in different sample areas in order to check surface homogeneity.

Optical-absorption spectra were recorded in the range of 250-800 nm on a Cary 5E (Varian) UV-Vis-NIR dual-beam spectrophotometer with a spectral bandwidth of $1 \mathrm{~nm}$. In each spectrum, the silica substrate contribution was subtracted.

Transmission electron microscopy (TEM) images were taken with a Philips 400T, operating at $120 \mathrm{kV}$. Ag/SiO specimens for TEM analyses were prepared under selected conditions on prethinned silica substrates (ca. $200 \mu \mathrm{m}$ thick). The substrates were subsequently ion milled to electron transparency using 6-kV $\mathrm{Ar}^{+}$ions, operating from the backside only and protecting the deposited surface from resputtering phenomena.

\section{RESULTS AND DISCUSSION}

The synthesis of $\mathrm{Ag} / \mathrm{SiO}_{2}$ nanosystems was performed under different conditions (Table I), with particular attention to the deposited silver amount as a function of the applied rf power $(W)$ and total pressure $(p)$. The combined influence of these two parameters was reflected by the value of the selfbias potential, $V_{\text {bias }}$ which is the dc potential developed on the target during plasma ignition (see Table I). ${ }^{33,36}$

$$
\left|V_{\text {bias }}\right| \propto\left(\frac{W}{p}\right)^{1 / 2} .
$$

The modulus of the self-bias potential versus the root square of the applied rf power is displayed in Fig. 1 for sets $1-3$, evidencing an optimal agreement with the theoretical behavior. In fact, apart from the linear increase of $V_{\text {bias }}$ with $W^{1 / 2}$ for each set of samples, higher self-bias potentials were observed on decreasing the total pressure, all the other parameters being constant, as expected from Eq. (1). As a gen- 


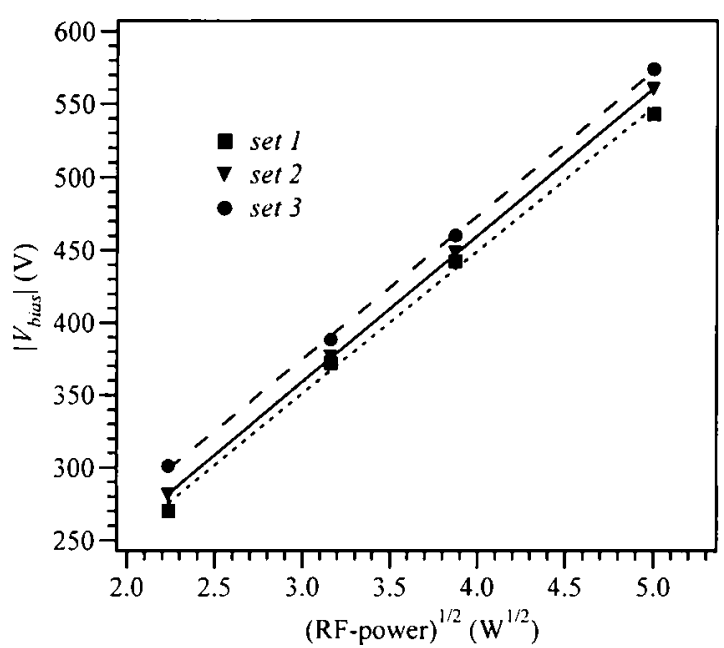

FIG. 1. Dependence of the self-bias potential $V_{\text {bias }}$ on the root square of the rf power for the synthesized sample sets.

eral rule, the tailoring of $V_{\text {bias }}$ by a proper choice of the rf power and total pressure is a valuable tool for the process control, since this parameter is proportional to the sputtering yield. ${ }^{37}$ Nevertheless, the knowledge of the self-bias potential does not in itself guarantee a thorough understanding of the synthesized $\mathrm{Ag} / \mathrm{SiO}_{2}$ nanosystem properties, since both homogeneous and heterogeneous processes must be taken into proper account.

In order to perform a real-time monitoring of Ag deposition processes, we employed a LRI system, recently developed and applied for the diagnostics of gold growth on $\mathrm{SiO}_{2} \cdot{ }^{33}$ As an example, the dependence of the reflectance $(R)$ on time for set 1 specimens is displayed in Fig. 2. A progressive increase of the LRI signal was evidenced as a function of time from $3.4 \%$ (the value for the bare silica substrate at the laser wavelength ${ }^{33}$ ) to a final value dependent on the applied rf power. Such behavior could be related to the progressive substitution of the ambience/substrate interface with the more reflecting ambience/silver one and, in particular, to

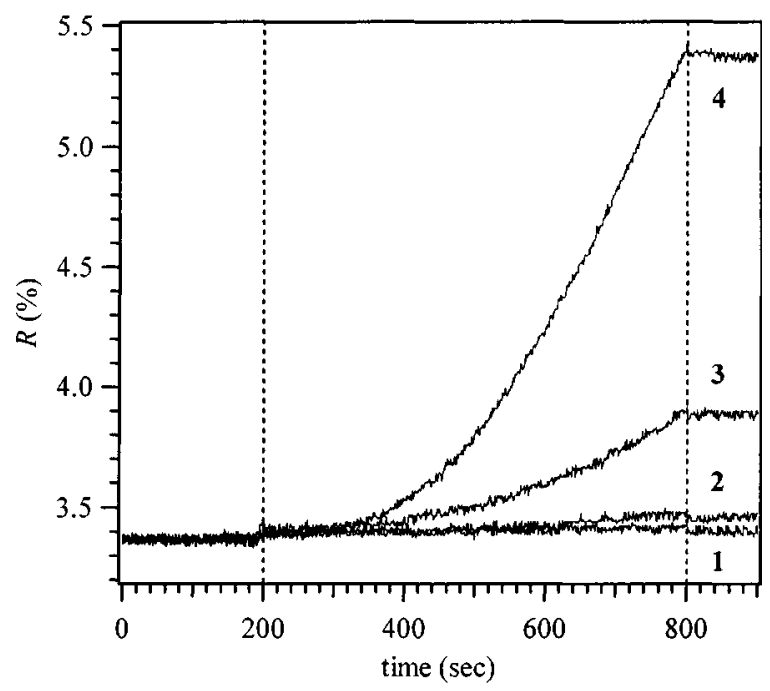

FIG. 2. Reflectance (\%) vs time traces obtained by LRI during Ag deposition on $\mathrm{SiO}_{2}$ for the set 1 specimens. The vertical lines mark the beginning (plasma on) and the end (plasma off) of the deposition process.

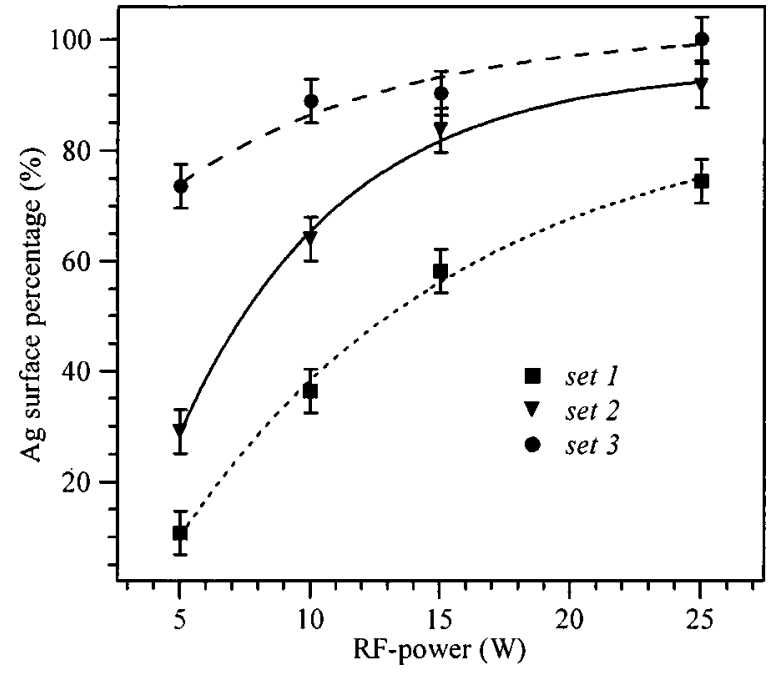

FIG. 3. Ag surface percentage as a function of the applied rf power for the three sample sets. The silver percentage was evaluated including only $\mathrm{Ag}$ and Si photopeaks.

the increase of the sputtering yield on going from sample 1 to 4 (i.e., from 5 to $25 \mathrm{~W}$ ). The same qualitative trend was observed in the case of all the synthesized sample sets, but resulted progressively steeper on lowering the total pressure. Such a result could be traced back to the increase of the sputtering yield on going from 0.38 to 0.23 and, finally, to $0.08 \mathrm{mbar}$, all the other parameters being constant, in agreement with Eq. (1). For samples with the lowest Ag amount (1 and 2), only small reflectance variations were observed (see Fig. 2) since scattering processes and optical losses were dominant due to the presence of highly dispersed nanoparticles. ${ }^{38}$ On increasing the substrate coverage, the progressive coalescence and agglomeration between particles resulted in an enhanced reflectance. ${ }^{38}$ Nevertheless, it is worth observing that no interference fringes in the LRI traces were ever detected even in the case of a complete substrate coverage. This phenomenon was attributed both to the low film thickness $(\leqslant 2 \mathrm{~nm})$ and to the low refraction index $(n$ $=0.140)$ and high extinction coefficient $(k=4.29)$ of Ag at $670 \mathrm{~nm} .{ }^{39}$ Moreover, even in the case of specimens characterized by a Ag surface percentage of $100 \%$ (see below, Fig. 3 , sample 12), LRI signals never reached reflectance values higher than $\approx 10 \%$, which are significantly lower than those expected for bulk silver (97.2\%). ${ }^{18,38,39}$ Such a phenomenon could be traced back to the peculiar morphology of the specimens, and, in particular, to the nanometric size of the deposited particles. ${ }^{8,18}$ Furthermore, the low deposited silver amount could be considered responsible for deviations from the optical properties of bulk Ag. ${ }^{8}$ The obtained results evidence the importance of LRI for both the real-time monitoring of the growth process and the obtainment of complementary information with respect to other ex situ techniques (see below).

The above results were confirmed by XPS characterization, evidencing an exponential increase of the $\mathrm{Ag}$ percentage with the rf power for each set of samples (Fig. 3). Moreover, lowering the total pressure at constant rf power resulted in higher deposited silver amounts, as expected for the in- 


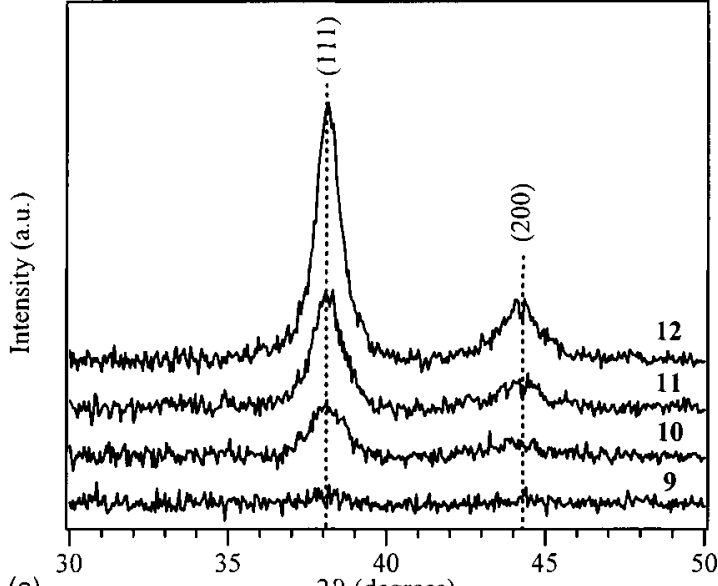

(a)

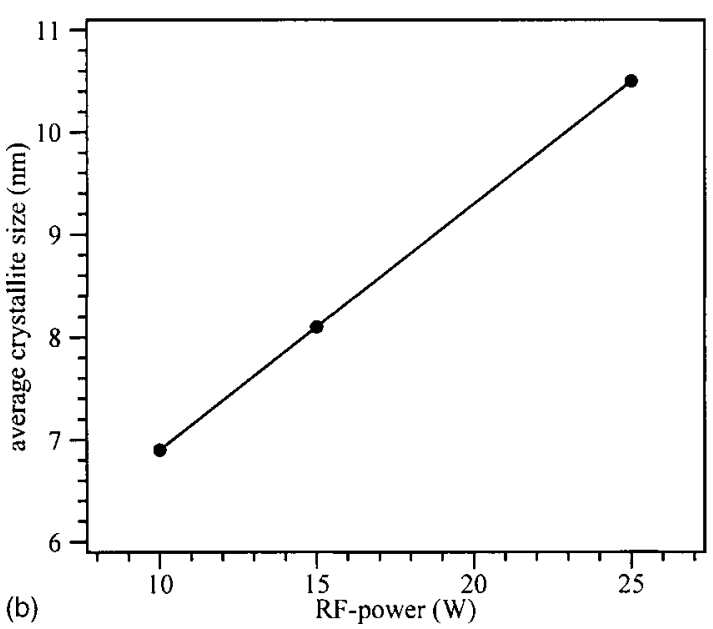

FIG. 4. (a) GIXRD patterns for the set 3 specimens. The vertical lines indicate the peak positions for bulk silver. ${ }^{40}$ (b) Average crystallite size vs rf power in the case of the same sample set.

crease of the sputtering yield. In other words, a suitable combination of the rf power and total pressure allowed us to control the Ag content resulting, in turn, in systems with tunable properties.

Further investigation was focused on the $\mathrm{Ag} / \mathrm{SiO}_{2}$ nanostructure, as probed by GIXRD. Selected spectra are displayed in Fig. 4(a) for specimens 9-12, evidencing the presence of the (111) and (200) reflections expected in the case of metallic $\mathrm{Ag}$ at $2 \vartheta=38.1$ and $44.3^{\circ}$, respectively. ${ }^{18,24,25,32,40}$ Unlike other literature reports, neither appreciable preferential orientations $s^{3,18,38}$ nor diffraction signals arising from silver oxides ${ }^{7,13}$ have ever been detected. A progressive intensity increase and narrowing of the peaks occurred when higher rf powers were applied. This effect could be ascribed both to a higher deposited Ag amount and to the enhanced crystallinity degree under more drastic plasma conditions. Concomitantly, a linear increase of the crystallite size with the rf power was observed [Fig. 4(b)], pointing out to the possibility of exerting a careful control on the system sizedependent properties. Such behavior, similar to that observed for $\mathrm{Au} / \mathrm{SiO}_{2}$ specimens, ${ }^{33}$ suggested that $\mathrm{Ag}$ growth on $\mathrm{SiO}_{2}$ was likely to occur by a preferential interaction of the impinging silver species with preexisting sites rather than with silica.
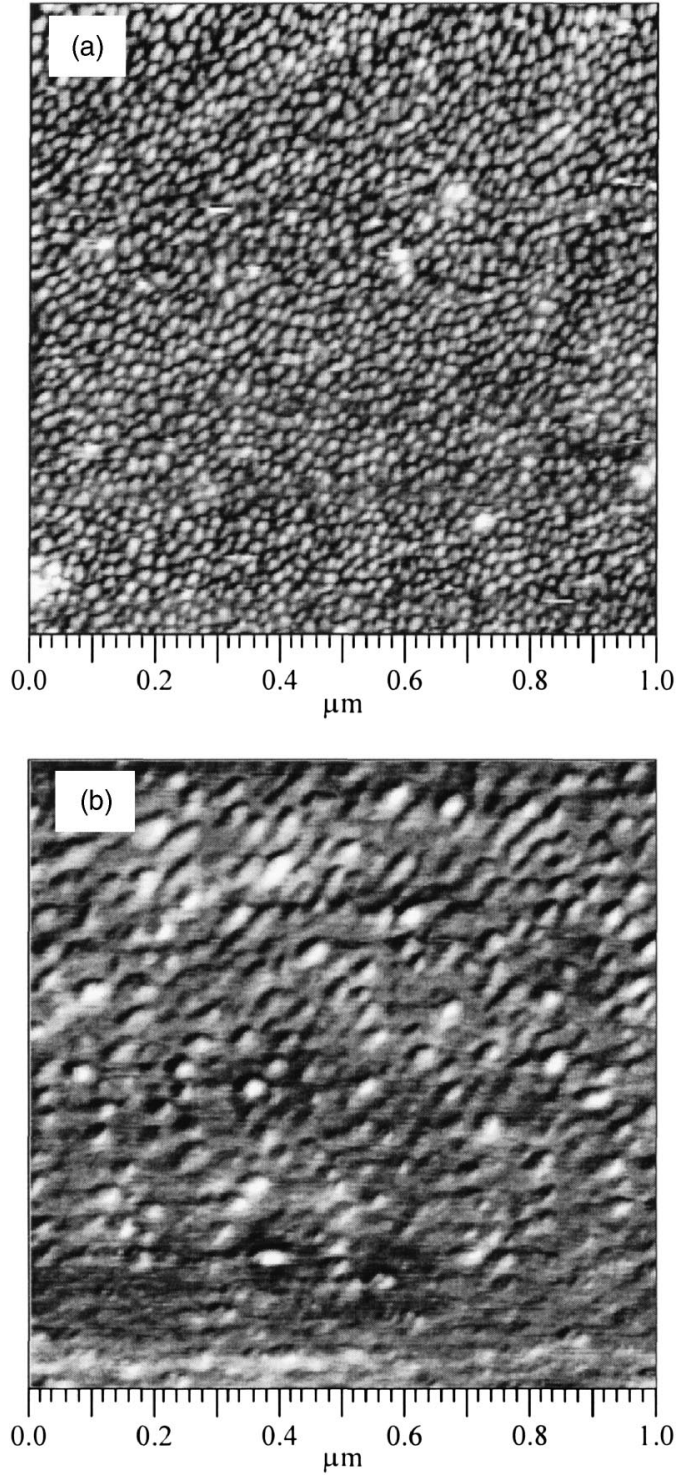

FIG. 5. Representative AFM micrographs for two selected specimens: (a) sample 4 (average grain size $\approx 14 \mathrm{~nm}$ ); (b) sample 12 (average grain size $\approx 45 \mathrm{~nm}$ ). In both cases, the vertical scale is from 0 to $10 \mathrm{~nm}$.

Surface morphology was investigated by AFM (Fig. 5). All specimens were characterized by a homogeneous and globular surface texture. Interestingly, a progressive increase of the particle size along with the deposited silver amount was observed, as expected in the case of a three-dimensional (Volmer-Weber) growth mode..$^{2,4,8,9,14}$ For all samples, the average grain size turned out to be larger than the average crystallite dimensions estimated by GIXRD, evidencing that the observed AFM particles were likely to be composed of several crystallites and/or some amorphous material.

The chemical composition as a function of processing conditions was analyzed by XPS and XE-AES. Despite the adoption of special precautions to minimize air exposure, interaction with the outer atmosphere could not be completely avoided, leading to the presence of chemical species different from the sole metallic silver. Such behavior, whose occurrence confirms the well-known complexity of the Ag-O system, ${ }^{41,42}$ reflected the silver reactivity towards oxygencontaining species, ${ }^{43}$ enhanced in the present case by the 

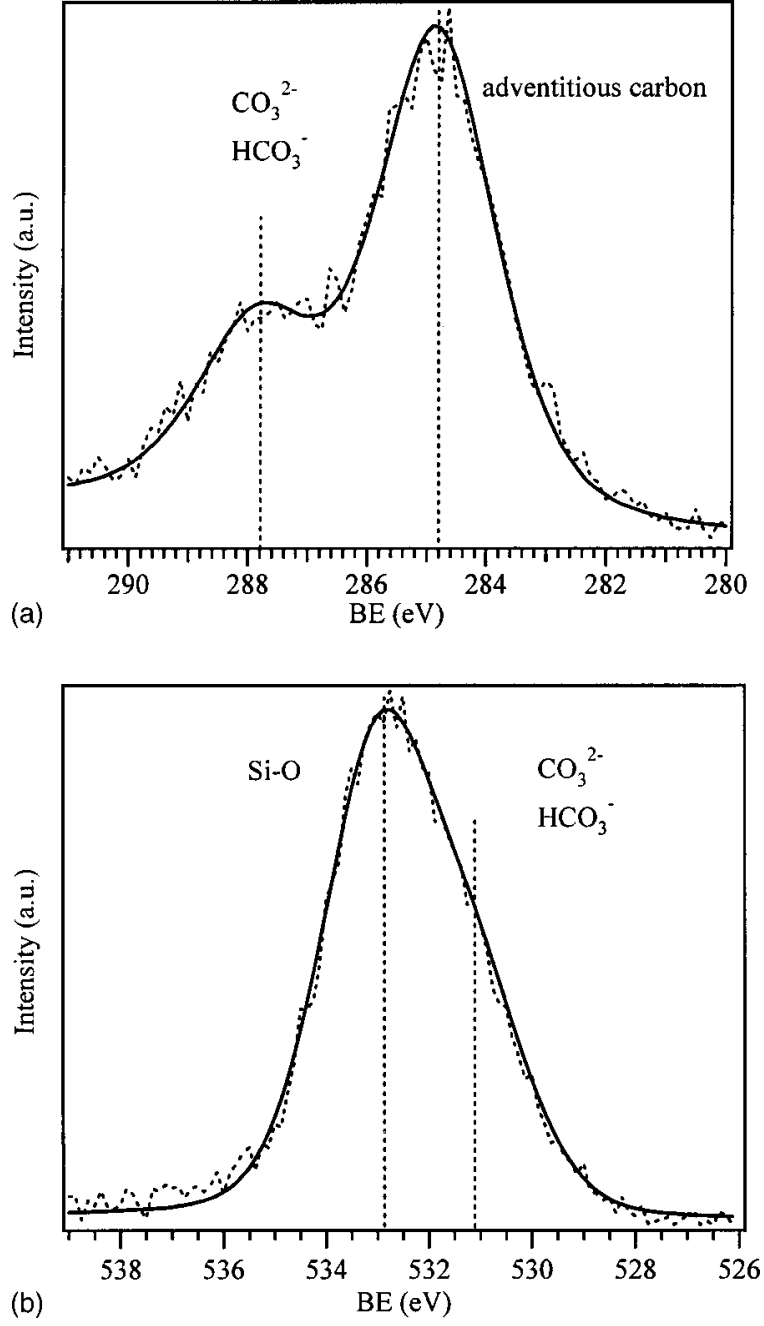

FIG. 6. C1s (a) and $\mathrm{O} 1 s$ (b) surface photoelectron peaks for sample 4.

system nanostructure. Such phenomena can be exemplified by the $\mathrm{C} 1 s$ and $\mathrm{O} 1 s$ spectra of specimen 4 (Fig. 6). In particular, the $\mathrm{C} 1 s$ photopeak [Fig. 6(a)] was characterized by two components: the main one $(\mathrm{BE}=284.8 \mathrm{eV})$, assigned to the presence of adventitious carbon, and a second one (BE $=287.8 \mathrm{eV}$ ), ascribed to carbonates or bicarbonates ${ }^{42,44}$ arising from atmospheric exposure.

Similar attributions were corroborated by the analysis of the O1s signal [Fig. 6(b)], characterized by a component at $\mathrm{BE} \approx 533.0 \mathrm{eV}$ due to the silica substrate and a lower $\mathrm{BE}$ peak at $\approx 531.1 \mathrm{eV}$ mainly ascribable to $\mathrm{Ag}_{2} \mathrm{CO}_{3} /$ $\mathrm{AgHCO}_{3} \cdot{ }^{42,44}$ However, other species could also be responsible for the latter band, including hydroxyl groups and adsorbed $\mathrm{H}_{2} \mathrm{O} / \mathrm{O}_{2} \cdot{ }^{41,42}$ As a general rule, both the $\mathrm{O} 1 s$ and the $\mathrm{C} 1 s$ signals showed a higher $\mathrm{CO}_{3}^{2-} / \mathrm{HCO}_{3}^{-}$contribution for samples characterized by a lower silver content. This effect could be explained taking into account that the latter specimens have a lower particle size (see above), thus being the most reactive to atmosphere due to their nanostructure. The atmospheric contamination was limited to the particle surface, as confirmed by the disappearance of $\mathrm{C}$ and $\mathrm{O}$ photoelectron signals after a mild $\mathrm{Ar}^{+}$sputtering.

The presence of oxidized $\mathrm{Ag}$ in the samples was confirmed by the calculation of silver Auger $\alpha$ paramet-
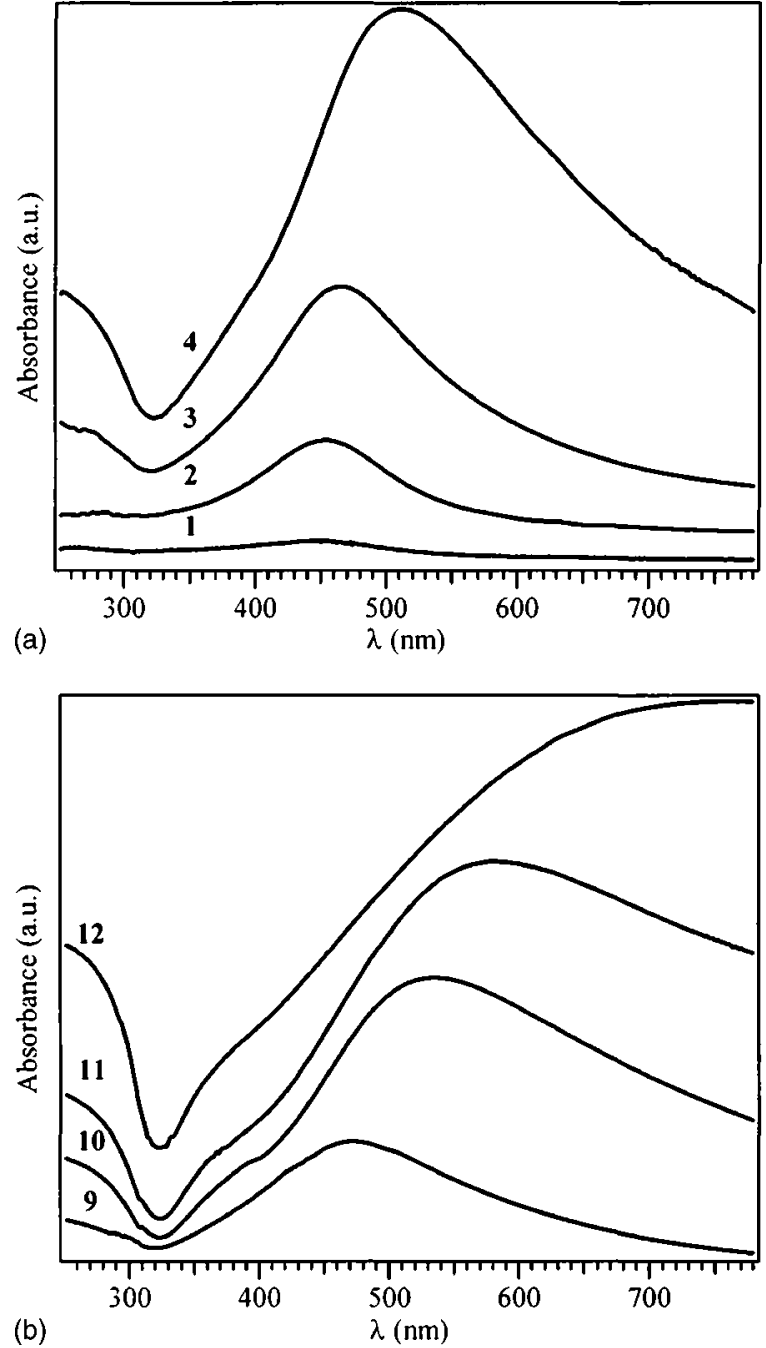

FIG. 7. Optical-absorption spectra for samples belonging to (a) set 1 and (b) set 3 .

ers $\quad\left[\alpha_{1}=\mathrm{BE}\left(\mathrm{Ag} 3 d_{5 / 2}\right)+\mathrm{KE}\left(\mathrm{M}_{5} \mathrm{NN}\right) ; \quad \alpha_{2}=\mathrm{BE}\left(\mathrm{Ag} 3 d_{5 / 2}\right)\right.$ $\left.+\mathrm{KE}\left(\mathrm{M}_{4} \mathrm{NN}\right)\right]^{24,45,46}$ This evaluation is necessary since the chemical shift of the Ag3d peak alone does not allow an unambiguous distinction among the various $\mathrm{Ag}$ chemical states. ${ }^{5,24}$ As a matter of fact, for all the synthesized specimens, the obtained values $\left(\alpha_{1}=720.0 \mathrm{eV} ; \alpha_{2}=725.4 \mathrm{eV}\right)$ fall in between $\operatorname{Ag}(0)$ and $\operatorname{Ag}(\mathrm{I})$ reported data. ${ }^{16,45}$

The presence of metallic silver was confirmed by optical-absorption spectra. In particular, the evolution of the system optical properties is displayed in Fig. 7 for sample sets 1 and 3. As concerns Fig. 7(a), three major effects could be detected on going from sample 1 to sample 4, i.e., on increasing the silver surface percentage from $\approx 10 \%$ to $\approx 75 \%$ (see Fig. 3): (i) the appearance and the progressive increase of an absorption band in the Vis region, attributable to the surface-plasmon resonance (SPR) of $\mathrm{Ag} / \mathrm{SiO}_{2}$ nanosystems; ${ }^{4,16,24,31}$ (ii) a concomitant broadening of the same signal; and (iii) a redshift from $\lambda \approx 450$ to $\approx 510 \mathrm{~nm}$ (compare Fig. 8).

The band intensity increase could be mainly traced back to the higher sputtering yield on increasing the rf power from 5 (sample 1) to $25 \mathrm{~W}$ (sample 4), thus resulting in the deposition of a progressively higher metal amount. In particular, 


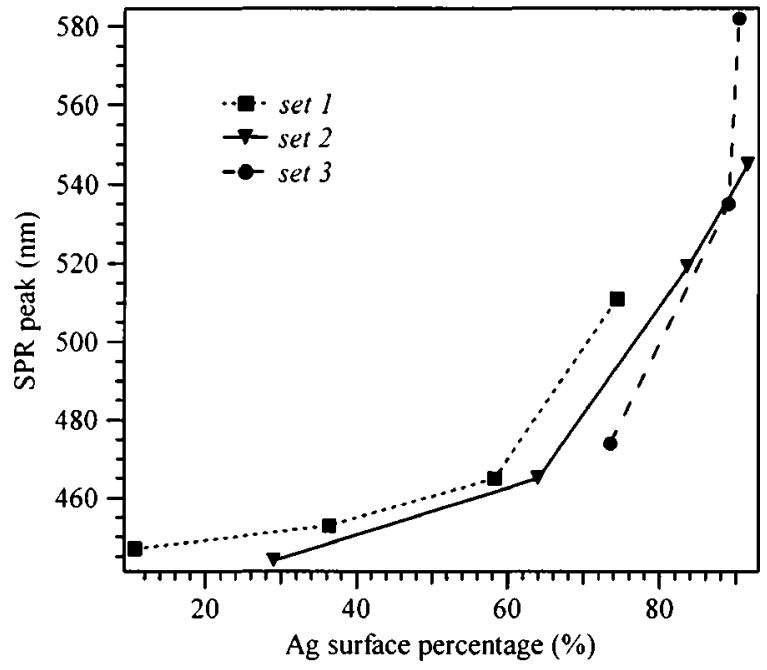

FIG. 8. Dependence of the SPR peak position on silver surface percentages obtained by XPS.

in the case of sample 1, the small Ag nanoparticle size was also responsible for the very weak absorption. ${ }^{1,24}$ Moreover, the observed peak broadening could be attributed to a gradually wider size and shape distribution when the silver percentage is increased. ${ }^{4,20,23}$ This phenomenon was likely to be responsible also for the marked peak asymmetry in the samples featuring a higher $\mathrm{Ag}$ content. ${ }^{25,31}$ Finally, the peak shift towards higher wavelengths could be ascribed to the absorption by larger coalescence aggregates ${ }^{16,22,23,25}$ and to the increasing interaction between more and more densely packed Ag nanoparticles. ${ }^{26}$

A more marked evolution of the optical properties was observed in the case of a higher Ag content, as evidenced for the set 3 specimens in Fig. 7(b). In fact, going from sample 9 $(\approx 75 \% \mathrm{Ag})$ to $11(\approx 90 \% \mathrm{Ag})$, the absorption spectra characterized by broader and more asymmetric bands, undergoing a progressive redshift (Fig. 8), were obtained. Finally, concerning specimen 12, no net band in the Vis region could be clearly discerned. Such a phenomenon was ascribed to the increasing interactions between previously isolated particles at higher silver amounts, ${ }^{8}$ reflecting the inter- and intraband electron excitations due to the continuous film structure, rather than those of discontinuous $\mathrm{Ag} / \mathrm{SiO}_{2}$ nanosystems displaying SPR bands. ${ }^{26}$ This conclusion is consistent with the XPS results, which showed complete silica coverage in the case of sample 12 (see below). In this case, the absorbance minimum at $\lambda \approx 320 \mathrm{~nm}$ closely resembles the so-called transmission window, already detected in the optical spectra of $\mathrm{Au} / \mathrm{SiO}_{2}$ nanosystems. ${ }^{33}$

In order to attain a deeper insight into the system nanostructure, selected specimens were subjected to TEM investigation, which evidenced a progressive transition from discontinous Ag nanosystems to continuous silver films when the deposited metal amount was increased.

In particular, sample 5 (Fig. 9, $\approx 30 \%$ Ag atomic percentage) was characterized by almost spherical and wellseparated particles uniformly distributed on the silica substrate, with average dimensions of $\approx 12 \mathrm{~nm}$ and a narrow size distribution. In the case of higher Ag amounts (sample 9

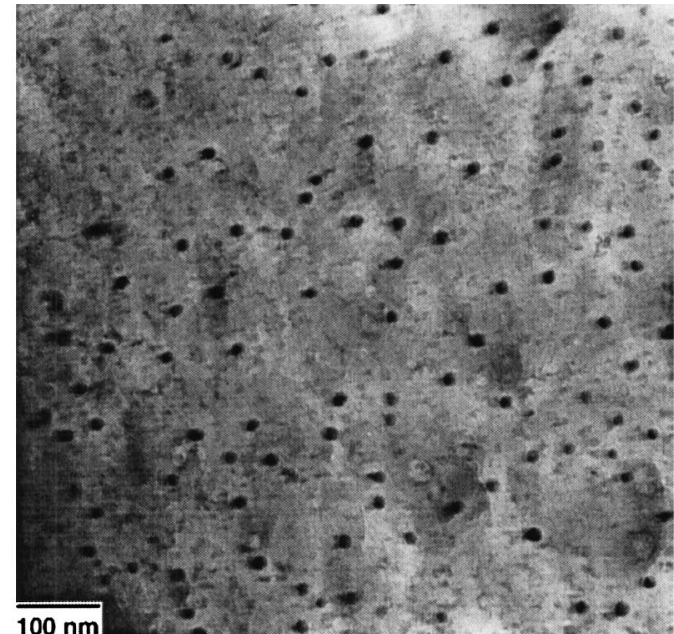

(a)

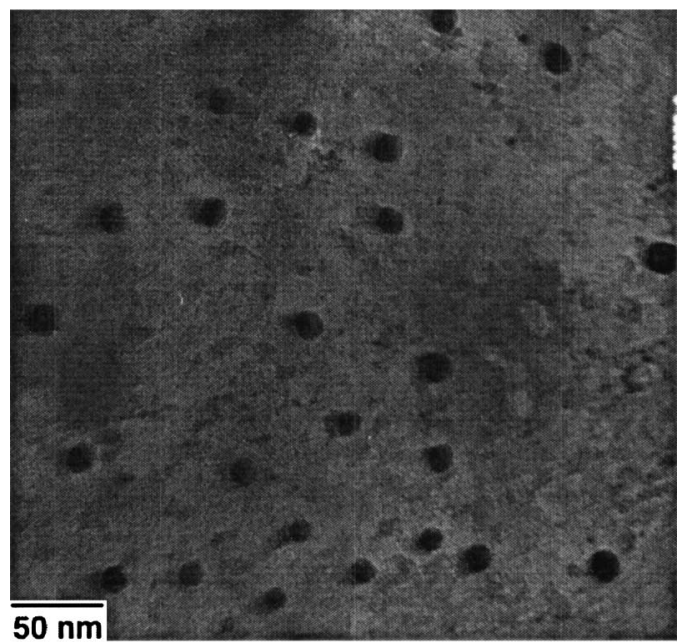

(b)

FIG. 9. Plane-view TEM images of specimen 5.

in Fig. 10, $\approx 75 \% \mathrm{Ag}$ ), a different morphology was observed. In fact, the images were dominated by a higher density of silver aggregates, resulting in a lower interparticle distance. Furthermore, several agglomerates showed marked variations from the spherical shape. The average particle size was higher than in the previous sample and corresponded to a broader size distribution $(10-50 \mathrm{~nm})$. It is worth highlighting that, as evidenced by the image contrast, most particles appeared to be composed of several crystallites ${ }^{3}$ and with structural planar defects, like twins. The corresponding selected area electron diffraction (SAED) pattern [Fig. 10(c)] was characterized by the presence of diffuse rings, confirming a random orientation for the crystalline domains and a low particle size.

In summary, the information gained by an accurate comparison of the above results allows a detailed insight into the growth and nucleation processes of $\mathrm{Ag}$ on $\mathrm{SiO}_{2}$, which can be explained as follows:

(1) At the beginning of the sputtering process $\mathrm{Ag}_{n} / \mathrm{Ag}_{n}^{+}$species impinge on the silica surface from the plasma phase, giving rise to very small and far apart nucleation sites. During the first stages, i.e., when the deposited 


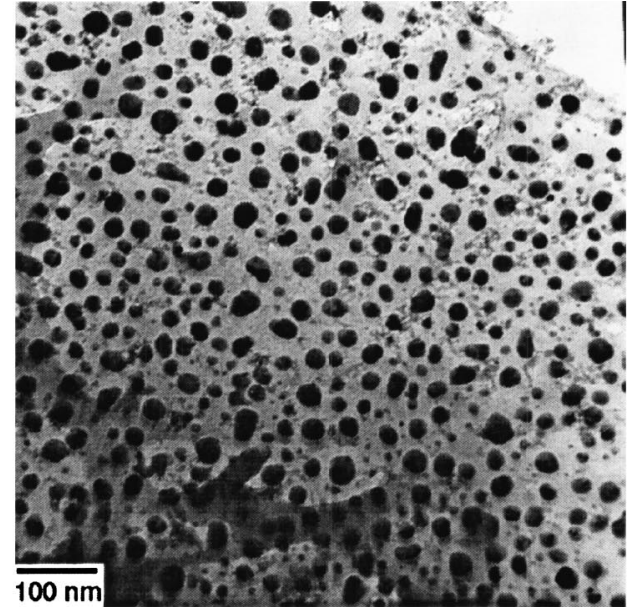

(a)

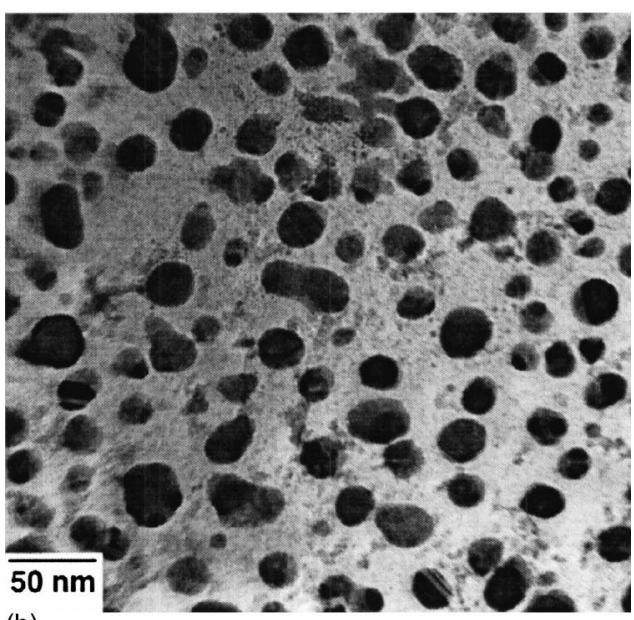

(b)

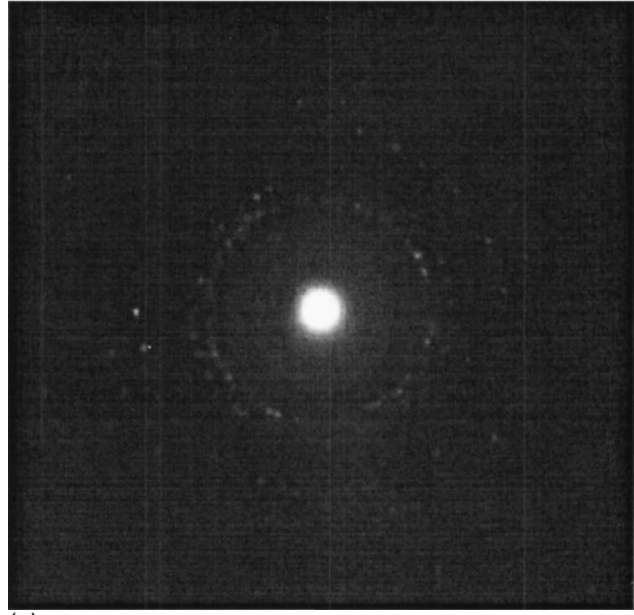

(c)

FIG. 10. Plane-view TEM images (a,b) and SAED pattern (c) of sample 9

silver amount is low enough, very small spherical nanoparticles, with a narrow size distribution, are formed on the silica surface. The very high surface-to-volume ratio, resulting in enhanced scattering phenomena, and the low metal content lead to a limited reflectance increase compared to the bare silica substrate and in a relatively weak SPR band. For the same reasons, no appreciable diffraction peaks are detected, even though the presence of silver-containing species is confirmed by XPS analyses.
(2) A progressive increase in the silver deposited amount results both in the formation of further nucleation sites and in the growth of preformed ones, according to a Volmer-Weber mechanism. It is interesting to note that these processes are accompanied by a marked evolution of the system properties. In fact, as the Ag percentage increases, bigger particles with a broader size distribution are typically observed. While some aggregates still appear spherical like, others present a prolate shape [cf. Fig. 10(a) and 10(b)]. All the above effects and the concomitant lowering of the interparticle distance result in a steeper increase of the system reflectance and in a stronger absorption band in the Vis region, whose intensity, shape, and position markedly depend on the system morphology.

(3) Finally, when the deposited metal amount is still higher, all particles are interconnected between each other to form a continuous layer which displays optical features different from discontinuous $\mathrm{Ag} / \mathrm{SiO}_{2}$ nanosystems.

\section{CONCLUSIONS}

In the present work, $\mathrm{Ag} / \mathrm{SiO}_{2}$ nanosystems were synthesized by rf sputtering from Ar plasmas at a substrate temperature as low as $60^{\circ} \mathrm{C}$. In particular, the influence of soft preparation conditions ( $\mathrm{rf}$ power $5-25 \mathrm{~W}$; Ar total pressure $0.08-0.38$ mbar) was investigated in terms of chemical composition, nanostructure, morphology, and optical properties. To this aim, beside the conventional ex situ characterization techniques (XPS, XE-AES, GIXRD, AFM, UV-Vis, and TEM), we adopted a LRI system for a real-time monitoring of $\mathrm{Ag}$ deposition processes on silica. No previous LRI reports on similar systems have ever appeared in the literature. A careful control of the substrate coverage, with a typical nanocrystal size $\leqslant 10 \mathrm{~nm}$ and average grain size up to $50 \mathrm{~nm}$, was attained. Finally, the progressive evolution from spherical and dispersed nanoparticles to bigger multidomain agglomerates and, ultimately, to continuous silver films as a function of the deposited metal amount, indicated the possibility of obtaining $\mathrm{Ag} / \mathrm{SiO}_{2}$ nanosystems with well-tailored structural and optical properties.

\section{ACKNOWLEDGMENTS}

The research program FISR-MIUR "Nanotecnologie molecolari per l' immagazzinamento e la trasmissione delle informazioni" is acknowledged for financial support. The authors are indebted to Dr. G. Bruno and Dr. M. Losurdo (IMIP-CNR, Bari, Italy) for their helpful suggestions.

${ }^{1}$ W. Cai, L. Zhang, H. Zhong, and G. He, J. Mater. Res. 13, 2888 (1998). ${ }^{2}$ Z. Pászti, G. Petö, Z. E. Horváth, A. Karacs, and L. Guczi, Solid State Commun. 107, 329 (1998).

${ }^{3}$ R. Chandra, P. Taneja, J. John, P. Ajjub, G. K. Dey, and S. K. Kulshreshtha, Nanostruct. Mater. 11, 1171 (1999).

${ }^{4}$ T. Wenzel, J. Bosbach, F. Stietz, and F. Träger, Surf. Sci. 432, 257 (1999). ${ }^{5}$ E. Traversa, M. L. Di Vona, P. Nunziante, S. Licoccia, T. Sasaki, and N. Koshizaki, J. Sol-Gel Sci. Technol. 19, 733 (2000).

${ }^{6}$ M. A. van Huis et al., Nucl. Instrum. Methods Phys. Res. B 191, 442 (2002).

${ }^{7}$ U. K. Barik, S. Srinivasan, C. L. Nagendra, and A. Subrahmanyam, Thin Solid Films 429, 129 (2003). 
${ }^{8}$ C. Charton and M. Fahland, Surf. Coat. Technol. 174-175, 181 (2003).

${ }^{9}$ C. Charton and M. Fahland, Vacuum 68, 65 (2003).

${ }^{10}$ W.-S. Ju, M. Matsuoka, K. Iino, H. Yamashita, and M. Anpo, J. Phys. Chem. B 108, 2128 (2004).

${ }^{11}$ S. Scirè, S. Minicò, C. Crisafulli, and S. Galvagno, Catal. Commun. 2, 229 (2001).

${ }^{12}$ N. Bogdanchikova, F. C. Meunier, M. Avalos-Borja, J. P. Breen, and A. Pestryakov, Appl. Catal., B, 36, 287 (2002).

${ }^{13}$ L. Gang, B. G. Anderson, J. van Grondelle, and R. A. van Santen, Appl. Catal., B, 40, 101 (2003).

${ }^{14}$ Y. Xiong, H. Wu, Y. Guo, Y. Sun, D. Yang, and D. Da, Thin Solid Films 375, 300 (2000).

${ }^{15}$ J. J. Mock, M. Barbie, D. R. Smith, D. A. Schultz, and S. Schultz, J. Chem. Phys. 116, 6755 (2002).

${ }^{16}$ G. De, A. Licciulli, C. Massaro, L. Tapfer, M. Catalano, G. Battaglin, C. Meneghini, and P. Mazzoldi, J. Non-Cryst. Solids 194, 225 (1996).

${ }^{17}$ E. Borsella et al., J. Non-Cryst. Solids 245, 122 (1999).

${ }^{18}$ T. Suzuki, Y. Abe, M. Kawamura, K. Sasaki, T. Shouzu, and K. Kawamata, Vacuum 66, 501 (2002).

${ }^{19}$ Y. Sarov, M. Nikolaeva, M. Sendova-Vassileva, D. Malinovska, and J. C. Pivin, Vacuum 69, 321 (2003).

${ }^{20}$ W. Cai, H. Hofmeister, and M. Dubiel, Eur. Phys. J. D 13, 245 (2001).

${ }^{21}$ W. Cai, H. Hofmeister, T. Rainer, and W. Chen, J. Nanopart. Res. 3, 443 (2001).

${ }^{22}$ K.-P. Charlè, L. König, S. Nepijko, I. Rabin, and W. Schulze, Cryst. Res. Technol. 33, 1085 (1998).

${ }^{23}$ H. Hövel, S. Fritz, A. Hilger, U. Kreibig, and M. Vollmer, Phys. Rev. B 48, 18178 (1993).

${ }^{24}$ L. Armelao, R. Bertoncello, and M. De Dominicis, Adv. Mater. (Weinheim, Ger.) 9, 736 (1997).

${ }^{25}$ L. Yang, Y. Liu, Q. Wang, H. Shi, G. Li, and L. Zhang, Microelectron. Eng. 66, 192 (2003).

${ }^{26}$ K. Murakoshi, H. Tanaka, Y. Sawai, and Y. Nakato, Surf. Sci. 532-535, 1109 (2003).

${ }^{27}$ A. L. Stepanov, D. E. Hole, and P. D. Townsend, Nucl. Instrum. Methods Phys. Res. B 166-167, 882 (2000).
${ }^{28}$ H. Tsuji, K. Kurita, Y. Gotoh, N. Kishimoto, and J. Ishikawa, Nucl. Instrum. Methods Phys. Res. B 195, 315 (2002).

${ }^{29}$ M. Adamik, P. B. Barna, and I. Tomov, Thin Solid Films 359, 33 (2000).

${ }^{30}$ H. C. Kim, T. L. Alford, and D. R. Allee, Appl. Phys. Lett. 81, 8287 (2002).

${ }^{31}$ M. Ferrari, L. M. Gratton, A. Maddalena, M. Montagna, and C. Tosello, J. Non-Cryst. Solids 191, 101 (1995).

${ }^{32}$ A. Rizzo, M. A. Tagliente, M. Alvisi, and S. Scaglione, Thin Solid Films 396, 29 (2001).

${ }^{33}$ D. Barreca, A. Gasparotto, E. Tondello, G. Bruno, and M. Losurdo, J. Appl. Phys. 96, 1655 (2004).

${ }^{34}$ V. Tsaneva, T. Donchev, R. Tomov, D. Ouzounov, A. Veneva, and T. Nurgaliev, Vacuum 48, 803 (1997).

${ }^{35}$ S. A. Catledge, P. Baker, J. T. Tarvin, and Y. K. Vohra, Diamond Relat. Mater. 9, 1512 (2000)

${ }^{36}$ Y. Catherine, in Diamond and Diamond-Like Films and Coatings, NATOASI Series B: Physics, edited by R. E. Clausing, L. L. Horton, J. C. Angus, and P. Koidl (Plenum, New York, 1991), Vol. 266, p. 193.

${ }^{37}$ J. E. Mahan, Physical Vapor Deposition of Thin Films (Wiley, Chichester, 2000).

${ }^{38}$ Y. S. Jung, Appl. Surf. Sci. 221, 281 (2004).

${ }^{39}$ D. W. Lynch and W. R. Hunter, in Handbook of Optical Constants of Solids, 1st ed., edited by E. D. Palik (Academic, Orlando, 1985), pp. 353-357.

${ }^{40}$ Pattern no 4-783, JCPDS (2000).

${ }^{41}$ J. S. Hammond, S. W. Gaarenstroom, and N. Winograd, Anal. Chem. 47, 2193 (1975).

${ }^{42}$ J. F. Weaver and G. B. Hoflund, J. Phys. Chem. 98, 8519 (1994).

${ }^{43}$ G. B. Hoflund, J. F. Weaver, and W. S. Epling, Surf. Sci. Spectra 3, 151 (1995).

${ }^{44}$ J. F. Weaver and G. B. Hoflund, Chem. Mater. 6, 1693 (1994).

${ }^{45}$ J. F. Moulder, W. F. Stickle, P. E. Sobol, and K. D. Bomben, in Handbook of X-Ray Photoelectron Spectroscopy, edited by J. Chastain (Perkin Elmer Corporation, Eden Prairie, MN, 1992).

${ }^{46}$ D. Briggs and M. P. Seah, Practical Surface Analysis (Wiley, Chichester, 1990), Vol. 1 